\title{
Micro-RNA-125a mediates the effects of hypomethylating agents in chronic myelomonocytic leukemia
}

\author{
Johannes Lorenz Berg ${ }^{1}$, Bianca Perfler ${ }^{1}$, Stefan Hatzl ${ }^{1}$, Marie-Christina Mayer ${ }^{1}$, Sonja Wurm', Barbara Uhl ${ }^{1}$, \\ Andreas Reinisch', Ingeborg Klymiuk², Sascha Tierling ${ }^{3}$, Gudrun Pregartner ${ }^{4}$, Gerhard Bachmaier ${ }^{4}$, \\ Andrea Berghold ${ }^{4}$, Klaus Geissler ${ }^{5,6}$, Martin Pichler ${ }^{7,8}$, Gerald Hoefler $^{9}$, Herbert Strobl $^{10}$, Albert Wölfler ${ }^{1}$, \\ Heinz Sill ${ }^{1}$ and Armin Zebisch ${ }^{1,11^{*}}$ (1)
}

\begin{abstract}
Background: Chronic myelomonocytic leukemia (CMML) is an aggressive hematopoietic malignancy that arises from hematopoietic stem and progenitor cells (HSPCS). Patients with CMML are frequently treated with epigenetic therapeutic approaches, in particular the hypomethylating agents (HMAs), azacitidine (Aza) and decitabine (Dec). Although HMAs are believed to mediate their efficacy via re-expression of hypermethylated tumor suppressors, knowledge about relevant HMA targets is scarce. As silencing of tumor-suppressive micro-RNAs (miRs) by promoter hypermethylation is a crucial step in malignant transformation, we asked for a role of miRs in HMA efficacy in CMML.

Results: Initially, we performed genome-wide miR-expression profiling in a Kras $^{G 12 D}$-induced CMML mouse model. Selected candidates with prominently decreased expression were validated by QPCR in CMML mice and human CMML patients. These experiments revealed the consistent decrease in miR-125a, a miR with previously described tumor-suppressive function in myeloid neoplasias. Furthermore, we show that miR-125a downregulation is caused by hypermethylation of its upstream region and can be reversed by HMA treatment. By employing both lentiviral and CRISPR/Cas9-based miR-125a modification, we demonstrate that HMA-induced miR-125a upregulation indeed contributes to mediating the anti-leukemic effects of these drugs. These data were validated in a clinical context, as miR125a expression increased after HMA treatment in CMML patients, a phenomenon that was particularly pronounced in cases showing clinical response to these drugs.
\end{abstract}

Conclusions: Taken together, we report decreased expression of miR-125a in CMML and delineate its relevance as mediator of HMA efficacy within this neoplasia.

Keywords: Chronic myelomonocytic leukemia, Hypomethylating agent, Azacitidine, miRNA, Tumor suppressor

\section{Background}

Chronic myelomonocytic leukemia (CMML) is an aggressive hematopoietic neoplasia, which is caused by malignant transformation of hematopoietic stem

*Correspondence: armin.zebisch@medunigraz.at

${ }^{1}$ Division of Hematology, Medical University of Graz, Auenbruggerplatz 38, 8036 Graz, Austria

Full list of author information is available at the end of the article and progenitor cells (HSPCs) [1]. It combines both myeloproliferative and myelodysplastic features and is therefore classified as a myelodysplastic syndrome/myeloproliferative neoplasia (MDS/MPN) overlap syndrome [1]. CMML has an inherent risk of acute myeloid leukemia (AML) transformation, particularly in cases classified as high-risk situations in the currently used risk-stratification tools $[2,3]$. Although some patients can be cured 
with high-dose chemotherapy and allogeneic hematopoietic stem cell (HSC) transplantation, the majority are diagnosed at an older age and therefore ineligible for this therapeutic approach $[2,3]$. The current gold standard for this patient collective are the hypomethylating agents (HMAs), azacitidine (Aza) and decitabine (Dec) $[3,4]$. HMAs act via inducing direct cytotoxicity on the one hand and via hypomethylation of DNA on the other hand. Particularly, DNA hypomethylation is thought to restore the expression of silenced tumor-suppressor genes, which in turn potentiates the anti-leukemic effects $[5,6]$. Unfortunately, the HMA target genes, which mediate the anti-leukemic effects of these drugs, are largely unexplored. Knowledge of these effectors could potentially help predict the success or even increase the efficacy of HMAs in CMML and other neoplasias.

Micro-RNAs (miRs) are small, noncoding RNA fragments and play a central role in the regulation of gene expression profiles [7, 8]. Aberrant expression profiles of miRs have been described in a broad selection of human tumors, and their functional relevance for malignant transformation is well established nowadays $[9,10]$. miRs have also been studied in myeloid neoplasias. In this context, they are functionally relevant for leukemogenesis on the one hand and for prognostic risk stratification on the other hand $[6,11,12]$. Of interest, the silencing of tumor-suppressive miRs in myeloid neoplasias is often caused by hypermethylation of the respective upstream/ promoter regions. Consequently, HMAs can reverse this miR downregulation in some cases, which has been shown to contribute to the anti-leukemic properties of these drugs [5, 6]. Unfortunately, however, the knowledge about miRs in CMML is scarce.

We hypothesized that the expression of tumor-suppressive miRs is decreased in CMML and that this downregulation is relevant for HMA treatment. Therefore, we performed miR expression profiling in murine and human CMML specimens and identified a consistent downregulation of the tumor-suppressive miR-125a. We could further show that decreased miR-125a expression is caused by hypermethylation of its upstream/ promoter region and can be restored by treatment with HMAs. Finally, we show that the anti-leukemic efficacy of these drugs is at least partly mediated via increasing the expression of miR-125a.

\section{Results}

miR-125a expression is decreased in CMML

We initially aimed to identify miRs with decreased expression in CMML. Therefore, we performed miR-microarray expression profiling, covering $1908 \mathrm{miRs}$, in a transgenic Kras $^{G 12 D}$-driven mouse model of CMML-like myeloproliferative disease (MPD) $[13,14]$. Although CMML is thought to arise from HSCs and early HSPCs, [15], we initially focused on $\mathrm{CD}-11 \mathrm{~b}^{-} / \mathrm{Ly}-6 \mathrm{G}^{-} / \mathrm{c}-\mathrm{Kit}^{+}$myeloid progenitor cells, which was a necessary step to produce sufficient amounts of RNA for microarray experiments (Fig. 1a). Interestingly, these analyses revealed extensive deregulation of miR expression profiles in myeloid progenitors of CMML-like MPD, with the majority showing decreased expression in the leukemia samples (Fig. $1 \mathrm{~b}$ and Table 1).

We then selected a series of miRs for further analyses according to the following criteria: i) the miR was amongst the TOP10 downregulated miRs and ii) a tumor-suppressive role was proven in myeloid leukemogenesis previously. Initially, we successfully validated the downregulation of the selected miR candidates (miR-125a, miR-150, and miR26a) [16-19] by qPCR (Fig. 1c). Next, we were interested in whether downregulation of these miRs truly occurs at the HSC level. In this respect, it has to be noted that we used $\mathrm{CD}-11 \mathrm{~b}^{-} / \mathrm{Ly}-6 \mathrm{G}^{-} / \mathrm{c}-\mathrm{Kit}^{+}$myeloid progenitors for our miRtranscriptome profiling in mice. Therefore, we additionally analyzed $\mathrm{Lin}^{-} / \mathrm{Sca}-1^{+} / \mathrm{c}-\mathrm{Kit}^{+}$(LSK) cells, which is a population enriched for HSCs [20]. The decreased expression of all three miRs could be validated within the LSK compartment (Fig. 1d), which indicates that their downregulation occurs at the HSC level.

We then were interested in whether these findings can be translated into human CMML. We, therefore, analyzed 36 primary CMML patient specimens (for clinical characteristics, see Additional file 1: Table 1) and compared the results to six healthy CD34 ${ }^{+}$HSPCs. In these assays, only miR125 a demonstrated a significantly decreased expression in the CMML specimens studied (Fig. 2a). It was previously shown that miR-125a is high in HSPCs and decreases during the physiologic myeloid differentiation [21]. As we compared whole bone marrow (BM) of CMML patients with a purified population of $\mathrm{CD} 34^{+}$HSPCs, our analysis might have been biased by the presence of differentiated cells within the CMML cases. To exclude such a scenario, we analyzed miR-125a expression in whole BM specimens of healthy donors and patients with lymphatic diseases without BM affection $(n=6)$. Again, miR-125a expression within these samples was significantly higher than in the CMML cases (Fig. 2b). Of note, decreased expression of miR-125a was not associated with a specific clinical or molecular feature of CMML (Additional file 1: Table 1, Additional file 1: Figs. S1-S2).

\section{Decreased miR-125a expression is caused by hypermethylation of its upstream/promoter region and can be increased by HMA treatment}

We then asked whether the decreased expression of miR$125 \mathrm{a}$ is caused by hypermethylation of its upstream/ promoter region. Recent work has pinpointed the role of hypermethylation of the miR-125a upstream region 


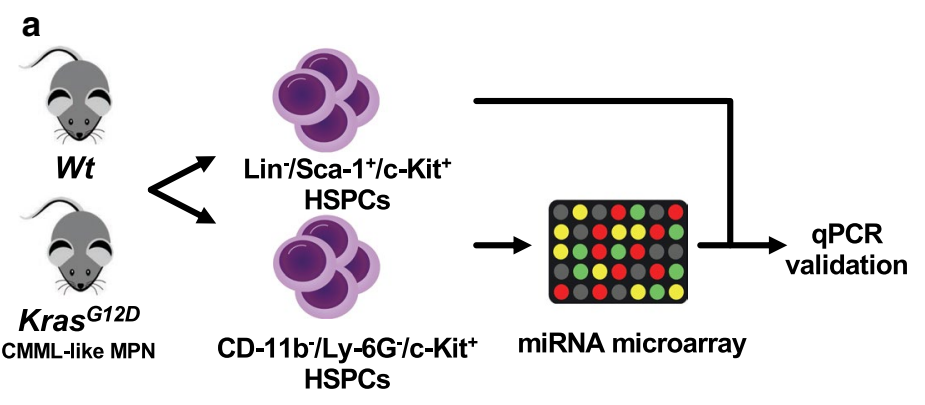

C

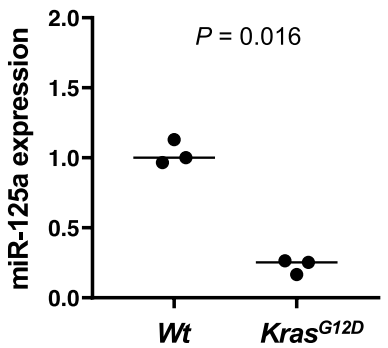

d

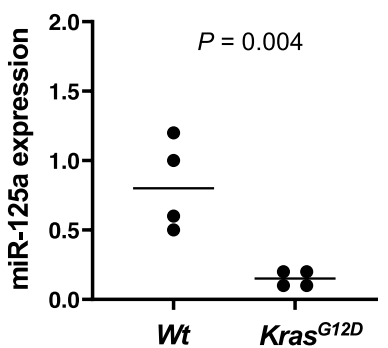

CD-11b-/Ly-6G-/c-Kit ${ }^{+}$

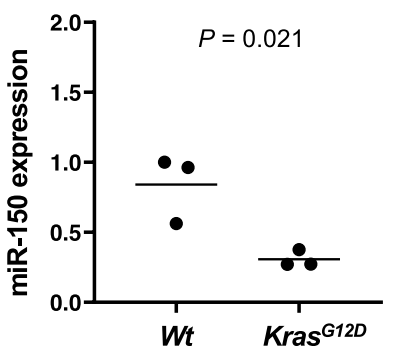

Lin $/$ Sca-1 ${ }^{+} / \mathrm{c}-\mathrm{Kit}^{+}$

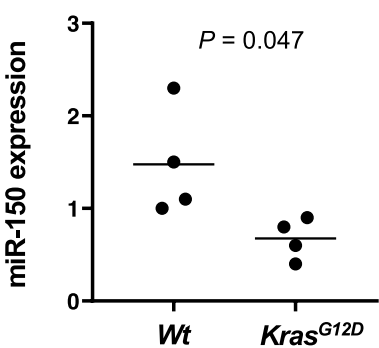

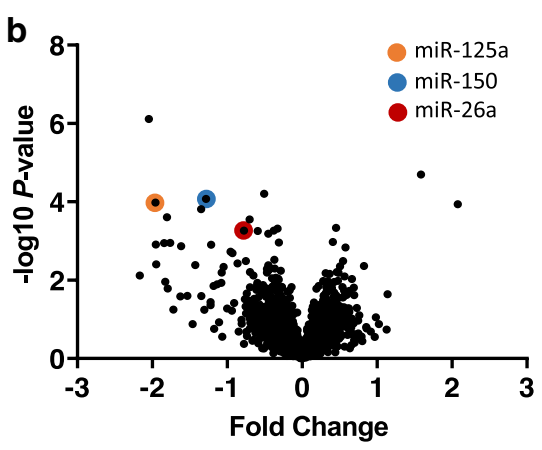
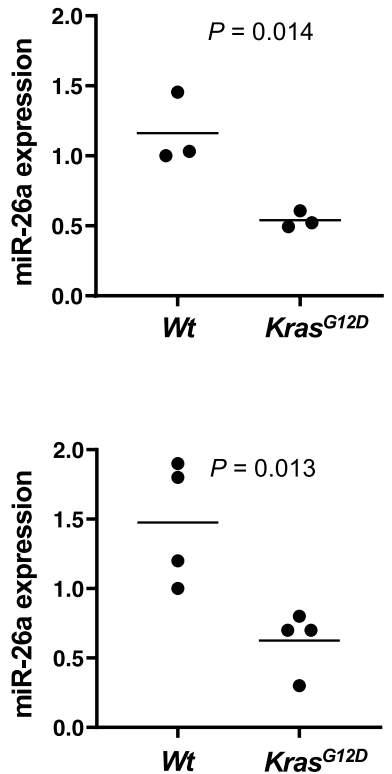

Fig. 1 Deregulation of miR expression levels in a murine model of CMML. a To screen for aberrant miR expression profiles in CMML, we performed miR-microarray analysis in CD-11 b-/Ly-6G-/c-Kit ${ }^{+}$HSPCs isolated from the bone marrow of mice with a Ras-induced CMML-like MPD. Therefore, four Mx1-Cre $/ \mathrm{Kras}^{\mathrm{G}}{ }^{12 \mathrm{D}}$ mice and four Kras ${ }^{W t}$ control mice were injected with plpC as outlined in the materials and methods section. Mice were killed and analyzed 6 weeks later. The development of the CMML-like MPD at this time was verified as outlined in Additional file 1: Fig S13. b Volcano plot showing deregulated miRs in CMML-HSPCs of $\mathrm{Mx1-Cre} / \mathrm{Kras}^{\mathrm{G}}{ }^{2 \mathrm{D}}$-mutated mice. The horizontal axis depicts the $x$-fold expression change in $\mathrm{M} \times 1-\mathrm{Cre}^{+} / \mathrm{Kras}^{G 12 D}$-mutated animals and transformed $P$-values are depicted on the vertical axis. miRs with higher expression in $\mathrm{Kras}^{G 12 D}$-mutated HSPCs are displayed on the right, whereas miRs with decreased expression levels in $\mathrm{Kras}^{G 12 D}$-mutated HSPCs are displayed on the left. c-d qPCR validation of the miR microarray results shows decreased miR-125a, miR-150, and miR-26a expression in CD-11 b $\mathrm{b}^{-} / \mathrm{Ly}^{-6 \mathrm{G}^{-}} / \mathrm{c}-\mathrm{Kit}^{+}(\mathbf{c}, \mathrm{n}=3)$ and $\mathrm{Lin}^{-} / \mathrm{Sca}-1^{+} / \mathrm{C}_{-} \mathrm{Kit}^{+}(\mathbf{d}, \mathrm{n}=4) \mathrm{HSPC}$ compartments of $\mathrm{Mx} 1-\mathrm{Cre}^{+} / \mathrm{Kras}^{G 12 D}$ mice. At least three mice per group were analyzed. The dot plots show the relative miR expression with the median indicated as horizontal line. One Kras ${ }^{W t}$ mouse was always used as a calibrator and its expression is set to 1. Group differences were assessed by t test. Wt, wildtype;

as the mechanism behind miR-125a downregulation [22-25]. To test this hypothesis, we employed a series of leukemia cell lines with decreased miR-125a expression (THP1, U937, NB4, Additional file 1: Fig. S3) and treated these cells with the HMAs Aza and Dec, respectively. Importantly, both substances significantly increased the expression of miR-125a in all cell lines tested (Fig. 3a,b, Additional file 1: Fig. S4). These data suggest that the decrease in miR-125a expression in myeloid leukemias is caused by hypermethylation and is reversible by
HMA treatment. To test this assumption, we performed bisulfite sequencing of the miR-125a upstream/promoter region in U937 cells before and after HMA treatment. Indeed, HMA treatment significantly decreased the methylation content within the upstream region studied (Fig. 3c). To delineate whether such an HMAinduced increase in miR-125a expression can also be observed in HMA-treated CMML patients, we analyzed the miR-125a expression in seven paired CMML patient specimens obtained before and after treatment with Aza 
Table 1 TOP10 downregulated miRs in CMML-MPDs of $\mathrm{Kras}^{G 12 D}$ mice. miRs were ranked according to their $x$-fold expression change in Kras ${ }^{612 D}$ mice as compared to the wildtype controls. Only statistically significant miRs with a mean signal intensity of $>4$ on all arrays were selected

\begin{tabular}{llll}
\hline Downregulated miRs & & & \\
\hline ID & Accession \# & Fold change & $\boldsymbol{p}$ value \\
\hline mmu-miR-99b-5p & MIMAT0000132 & -4.13 & $<0.001$ \\
mmu-miR-125a-5p & MIMAT0000135 & -3.89 & $<0.001$ \\
mmu-miR-130a-3p & MIMAT0000141 & -3.59 & 0.001 \\
mmu-miR-146a-5p & MIMAT0000158 & -3.49 & $<0.001$ \\
mmu-miR-99a-5p & MIMAT0000131 & -3.06 & 0.001 \\
mmu-miR-676-3p & MIMAT0003782 & -2.55 & $<0.001$ \\
mmu-miR-150-5p & MIMAT0000160 & -2.44 & $<0.001$ \\
mmu-let-7e-5p & MIMAT0000524 & -2.32 & 0.001 \\
mmu-miR-26a-5p & MIMAT0000082 & -1.71 & 0.001 \\
mmu-miR-3535 & MIMAT0031410 & -1.63 & $<0.001$ \\
\hline
\end{tabular}

or Dec. In agreement with our in vitro data, we thereby observed a statistically significant increase in miR-125a expression after HMA treatment (Fig. 4a). Interestingly, the increase in miR-125a expression was particularly pronounced in the five patients presenting with clinical response to HMA therapy (Fig. 4b; response assessment according to the recently published response criteria for MDS/MPN in adults [26]). Unfortunately, bisulfite sequencing in patient specimens failed due to technical reasons. Therefore, we performed a database analysis via the National Center for Biotechnology Information (NCBI)'s Gene Expression Omnibus (GEO; https ://www.ncbi.nlm.nih.gov/geo/; GSE40870). In this study, the authors performed genome-wide DNA methylation profiling in myeloid leukemia patient specimens treated with Dec and cytarabine, respectively, and compared the results to control-treated samples [27]. We re-analyzed the methylation of a specific CpG-site within the miR125 a upstream/promoter region and analyzed the methylation change to control-treated samples. Importantly, Dec caused significant demethylation of the miR-125a upstream/promoter region, whereas the classical chemotherapeutic agent cytarabine failed to do so (Additional file 1: Fig. S5). These data further support our observation that the HMA-induced increase in miR-125a expression is caused by demethylation of its upstream/promoter region.

\section{The anti-leukemic effects of HMAs are partly mediated} by increasing the expression of miR-125a

As described above, clinical data indicate that HMA treatment increases the expression of downregulated miR-125a in CMML patients and suggest a potential link between the rise of miR-125a and the clinical efficacy of HMAs. Therefore, we aimed to delineate whether some anti-leukemic HMA effects might be mediated via the increase in miR-125a expression. We initially tested the efficacy of Aza, the most employed HMA, in the abovementioned monocytic leukemia cell lines. In agreement with the published literature, this caused the induction of cell death, which was mediated via an increase in apoptosis (Additional file 1: Figs. S6-S7) [28, 29]. Aza thereby mimicked the effects of miR-125a overexpression, which increased the apoptosis within these cells as well (Additional file 1: Fig. S8). We then employed THP1 cells with a hairpin inhibitor-mediated knockdown of miR-125a (Additional file 1: Fig. S9). As this knockdown had been established by antisense oligonucleotides, it cannot be rescued by the treatment with HMAs. Indeed, Aza failed a

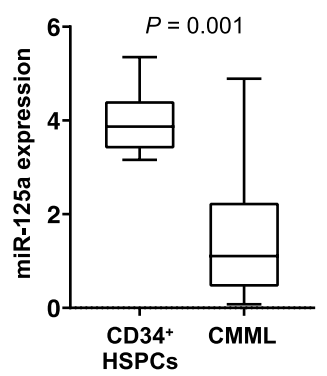

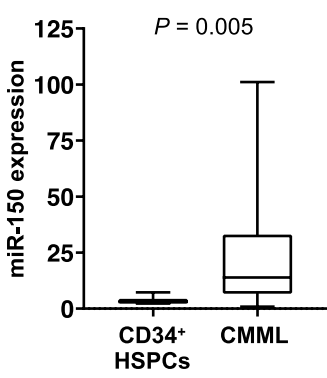

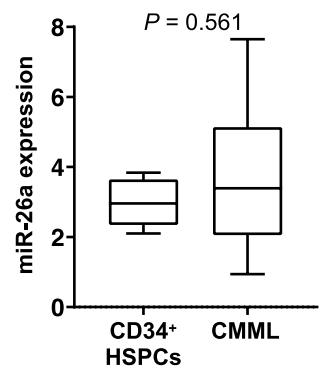

b

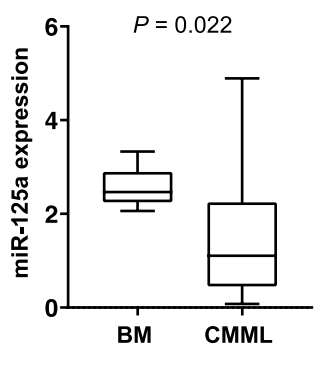

Fig. 2 miR-125a expression is decreased in primary CMML patient specimens. a The box plots show the relative miR-125a, miR-150, and miR-26a expression levels in 36 CMML patient specimens compared to six CD34+ HSPCs. $\mathbf{b}$ The relative expression level of miR-125a in 36 CMML patients compared to six whole BM specimens of healthy donors and patients with lymphatic diseases without BM affection is displayed. The graphs denote the miR expression normalized to a calibrator, set to 1 (U937 for miR-125a and miR-26a; GDM-1 for miR-150). Differences between the groups were assessed with the Mann-Whitney U test. CMML, chronic myelomonocytic leukemia; BM, bone marrow; HSPCs, hematopoietic stem and progenitor cells 


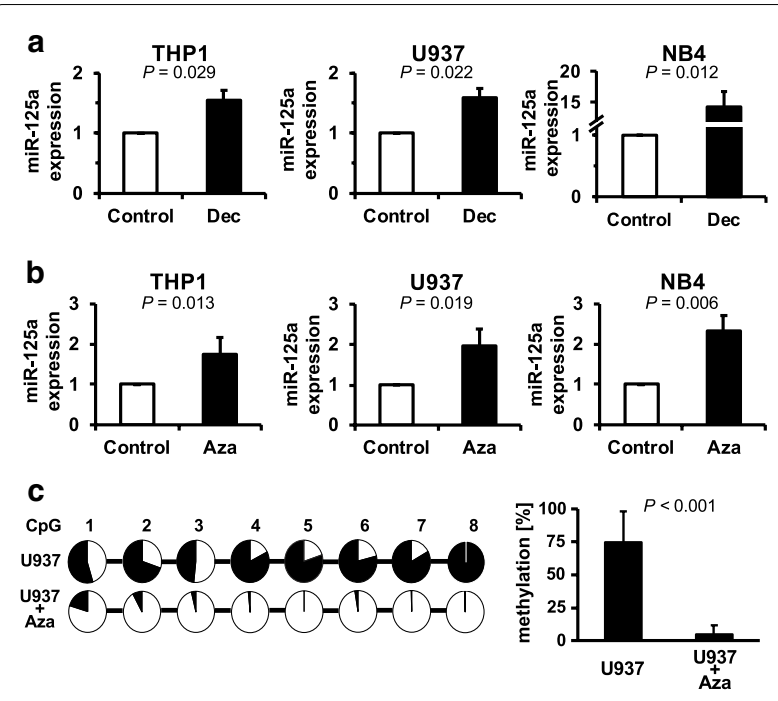

Fig. 3 Decreased miR-125a expression is caused by

hypermethylation of its upstream/promoter region and can be increased by HMA treatment. a miR-125a expression was assessed by qPCR in the myeloid cell lines THP1, U937, and NB4 after treatment with $5 \mu \mathrm{M}$ decitabine for $48 \mathrm{~h}$. For comparison of the different conditions, respective control situations (treated with the empty dissolvent only) were set at a value of 1 . The relative increase in miR-125a expression in the decitabine-treated conditions was calculated as the ratio of decitabine-treated to control-treated expression levels. $\mathbf{b}$ miR-125a expression after treatment with $2.5 \mu \mathrm{M}$ azacitidine for $24 \mathrm{~h}$. The relative increase in miR-125a expression in the azacitidine-treated conditions compared to controls is displayed. Graphs denote the mean \pm SD of at least three independent experiments. Comparisons against the control condition were performed using a one-sample t test against a reference value of 1 . Of note, the increase in miR-125a expression could also be observed after incubation with a lower azacitidine concentration ( $1 \mu \mathrm{M}$; Additional file 1: Fig. S4) c Eight CpG-sites within a CpG-rich region upstream of miR-125a [23] were studied by bisulfite sequencing in U937 cells before and after treatment with $2.5 \mu \mathrm{M}$ azacitidine for $24 \mathrm{~h}$. This region roughly correlates to the miR-125a promoter region [52]. The panel on the left demonstrates the percentage of methylated sequencing reads for each $\mathrm{CpG}$ site studied; the panel on the right depicts the mean percentage of methylation across all eight CpG sites \pm SD. Statistical differences were assessed with the $t$ test. Aza, azacitidine; Dec, decitabine

to increase the expression of miR-125a in these cells (Additional file 1: Fig. S10). Most importantly, the effects of Aza on apoptosis were significantly diminished in this situation (Fig. 5a), which indicates that, indeed, some anti-leukemic effects of HMAs are mediated by increasing miR-125a expression. To further corroborate these results, we repeated these experiments in U937 cells. As hairpin inhibitor-mediated miR-125a knockdown was unsuccessful in this cell line, we completely deleted miR125a by employing the CRISPR/Cas9 technology (Additional file 1: Fig S9). In agreement with the results from THP1, miR-125a deletion reduced the sensitivity to Aza

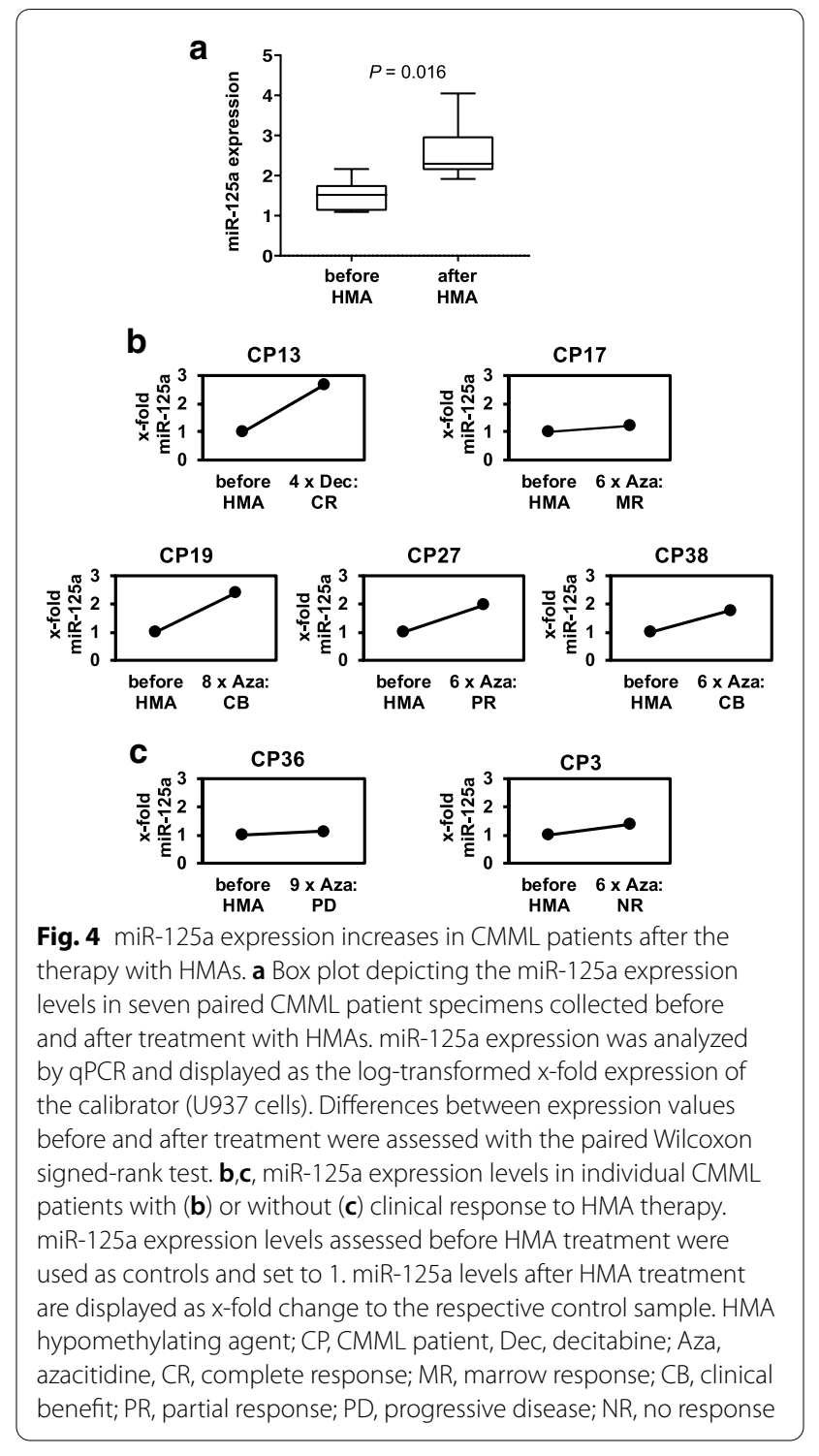

(Fig. 5b). Finally, we also tested the effects of Aza treatment in THP1 cells, where miR-125a expression was already increased by stable transfection with a miR-125a expression construct. In this situation, Aza treatment increased the miR-125a expression further, suggesting a synergistic or additive effect (Additional file 1: Figure S11). Indeed, Aza treatment in miR-125a-overexpressing cells caused a further increase in apoptosis when compared with miR-125a-overexpressing cells treated with empty dissolvent (Additional file 1: Figure S12).

\section{Discussion}

In this study, we aimed to delineate the potential mediators of HMA treatment response in CMML. We initially screened for a potential downregulation of 


\section{a}

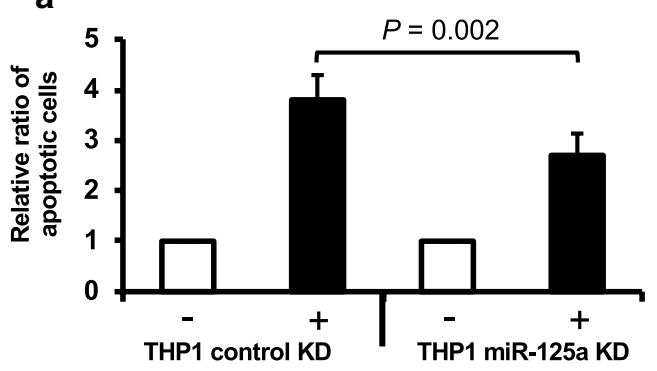

b

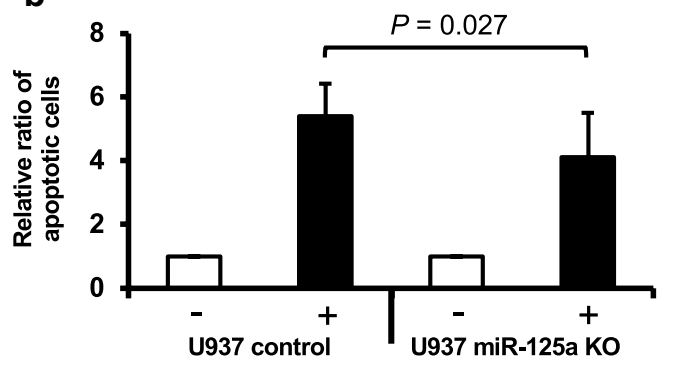

Fig. 5 The anti-leukemic effects of HMAs are partly mediated by increasing the expression of miR-125a. a THP1 cells were transiently transfected with a miR-125a-specific hairpin inhibitor (THP1 miR-125a KD) and scrambled control (THP1 control KD), respectively. b miR-125a was deleted in U937 by employing the CRISPR/Cas9 technology (U937 miR-125a KO); parental U937 cells were used as controls. Apoptosis was measured after 24-h treatment with Aza (2.5 $\mu \mathrm{M}$ for THP1, $5 \mu \mathrm{M}$ for U937; indicated as +) or empty dissolvent (indicated as -) by Annexin-V/7AAD assays. The respective control situations (treated with the empty dissolvent only) were set at a value of 1, and the relative increase in apoptosis in the Aza-treated conditions was calculated as the ratio of Aza-treated to control-treated cells. The graphs represent the mean \pm SD of at least three independent experiments. Statistical significance was evaluated using a paired Student's $t$ test

tumor-suppressive miR expression profiles in CMML by applying a miR-microarray platform covering more than 1900 miRs. By employing a genetically homogenous Kras $^{G 12 D}$-induced murine model of CMML, we were able to identify extensive deregulation of miR expression profiles, with the majority of these miRs being downregulated in CMML-HSPCs. By additionally analyzing 36 primary CMML patient specimens, we were able to validate miR-125a as one of the most prominently downregulated miRs in CMML, underlining the relevance of our mouse model for the human setting. Aberrant expression of miR-125a has been described in a variety of myeloid malignancies and proved to exhibit a tumor-suppressive function during myeloid leukemogenesis [16, 30]. Indeed, transgenic deletion of miR-125a in mice caused the development of MPDs and urogenital abnormalities [16]. These data are corroborated by in vitro data of the current study, where miR-125a overexpression in monocytic leukemia cells with decreased endogenous miR-125a had a pronounced anti-leukemic effect (Additional file 1: Fig S8). Interestingly, Tatsumi and co-workers reported that mice with a complete deletion of miR-125a developed a less severe MPD phenotype than animals with a heterozygous deletion; the latter showing a decreased but still measurable miR-125a expression. The authors thereby demonstrated compensatory inhibitory pathways that only became active when a complete deletion of miR-125a occurred. The role of miR-125a is further complicated by the fact that oncogenic properties were described in the context of myeloid leukemogenesis as well $[21,31]$. Taken together, the currently available functional in vivo data imply that miR-125 has to be finely tuned to keep HSPCs in a physiologic state and that miR125 a deregulation, both by increased and by decreased expression levels, may cause the development of myeloid leukemias. Such a scenario has also been shown for other miRs, including miR-126a, where both overexpression and knockdown enhanced murine AML1-ETO/RUNX1RUNX1T1-driven leukemogenesis [32]. To put these findings in a clinical context, it is necessary to delineate the expression profile of these miRs in primary patient specimens of the respective malignancy. In this study, we now report significantly decreased miR-125a in 36 primary patient specimens of CMML, which suggests that a decrease in miR-125a expression represents the major mechanism of miR-125a deregulation during CMML development and highlights its tumor-suppressive function within this malignancy.

Another interesting finding of our study was that decreased expression of miR-125a is caused by hypermethylation of its upstream/promoter region. These data are in line with results from AML and solid cancers [22-25]. Indeed, we demonstrated that miR-125a expression in myeloid cell lines with low endogenous miR-125a levels was significantly increased by treatment with both Aza and Dec. These findings are also in agreement with data from the primary patient specimens. By qPCRbased miR expression analyses in paired patient specimens obtained before and after/during HMA treatment, we observed that miR-125a expression levels after HMA therapy are significantly higher than before. Interestingly, we observed that the degree of miR-125a increase in HMA-treated patients was particularly pronounced in cases where HMAs demonstrated clinical efficacy. It has to be noted that this clinical observation is limited by the fact that our cohort of sequential samples was too small to corroborate these findings statistically. Additionally, the HMA overall response rate of $>70 \%$ within these cases seems to be rather high compared with results from the literature [3]. Hence, a selection bias of these 
cases cannot be excluded. While larger and prospective cohorts will be necessary to ultimately unravel a potential link between HMA-induced miR-125a increase and HMA efficacy, such an association has been described in a previous publication as well. Solly and co-workers studied miR expression profiles in high-risk MDS (HR-MDS) patients treated with Aza [33]. While they described a decreased expression of miR-125a in BM samples of HR-MDS patients as compared with healthy donors at diagnosis, they also described changes in miR-125a expression during Aza treatment. As seen in our study, the authors observed that miR-125a expression increased the following Aza treatment in patients with a sustained response, whereas the opposite was true for patients with primary or secondary Aza resistance. Based on these data, one might speculate that Aza has the potential to increase the expression of miR-125a and that this miR$125 \mathrm{a}$ increase might mediate some of the anti-leukemic effects of these substances. To test this assumption, we treated monocytic leukemia cells with Aza and assessed the anti-leukemic effects by measuring the induction of apoptosis. We then performed additional inactivation of miR-125a by shRNA-mediated knockdown and CRISPR/ Cas9-mediated knockout, respectively. This miR-125a silencing inhibited the Aza-induced increase in miR-125a expression. Most importantly, the prevention of miR125a upregulation reduced the effects of Aza on inducing apoptosis. These data indicate that at least some of the effects of HMAs are mediated via increasing the expression of miR-125a. Finally, it is worth mentioning that Aza treatment, combined with lentiviral miR-125a overexpression, had a synergistic/additive effect in our experiments. This finding is of particular relevance as the development of miR-mimic pharmaceutics is nowadays feasible [34]. It is appealing to imagine a situation where miR-125a mimics might be used to increase the efficacy of HMAs in CMML or to overcome the resistance to these drugs. While this scenario is a very optimistic outlook into a more distant future, our experiments warrant further preclinical work in this direction.

\section{Conclusions}

In conclusion, we describe a biologically relevant decrease in miR-125a expression in CMML, which is caused by hypermethylation of its upstream/promoter region. We further show that the treatment with HMAs can reverse miR-125a downregulation and that at least some of the anti-leukemic efficacy of HMAs is mediated via increasing the expression of miR-125a. These data provide more insight into the mode of action of HMAs in CMML and will aid in optimizing HMA treatment approaches for patients with myeloid malignancies.

\section{Methods}

\section{Primary CMML patient samples and cell lines}

CMML patient samples (peripheral blood or BM), as well as BM specimens from healthy donors and patients with lymphatic diseases without BM infiltration, were collected and stored at the Division of Hematology, Medical University of Graz (MUG), Austria. Additionally, CMML patient specimens from the Austrian Biodatabase for CMML $[4,35]$ were included in this study. All samples were processed and stored as described in detail previously [36-38]. All samples contained at least $80 \%$ myelomonocytic cells as determined by GiemsaMay-Grünwald-stained cytospin preparations. Healthy CD34+ HSPCs were collected from umbilical cord blood specimens (EasySep, STEMCELL Technologies, Vancouver, Canada) as described in detail previously [39]. $293 \mathrm{~T}$, U937, THP1, and NB4 cell lines were obtained from the German National Resource Center for Biological Material (DSMZ, Braunschweig, Germany) and the Core Facility Alternative Biomodels and Preclinical Imaging (Center for Medical Research at the MUG), respectively. Low passage stocks were frozen, and cells were cultivated for less than 6 months after thawing. Moreover, cells were screened by variable number of tandem repeat profiling (VNTR) for authenticity as previously described $[39,40]$.

\section{Mouse strains and isolation of murine HSPCs}

All mouse experiments were performed on a C57BL/6 strain background. Mice with a $\mathrm{M} x 1-\mathrm{Cre}^{+} / \mathrm{Kras}^{\mathrm{G12D}}$ genotype and wildtype controls were used for miRNA expression screening. Intraperitoneal injections of polyinosinic polycytidylic acid (pIpC, Sigma-Aldrich, St. Louis, MO, USA; three times $250 \mu \mathrm{g} / \mathrm{d}$ at every alternate day, starting at the age of 30 days) were performed in all mice. For miR expression analysis, mice were killed by cervical dislocation after anesthesia with isoflurane (4\%; AbbVie, North Chicago, IL, USA, mixed with 1,5 l/ min $\left.\mathrm{O}_{2}\right) 6$ weeks after pIpC injection. CMML-like MPD in $\mathrm{Kras}^{G 12 D}$-mutated animals was fully developed at this time (Additional file 1: Fig. S13). For miR-microarray and qPCR experiments, $\mathrm{Lin}^{-} / \mathrm{Sca}-1^{+} / \mathrm{c}^{-\mathrm{Kit}^{+}} \mathrm{HSCs}$ and CD$11 \mathrm{~b}^{-} / \mathrm{Ly}-6 \mathrm{G}^{-} / \mathrm{c}-\mathrm{Kit}^{+}$myeloid progenitor cells, respectively, were collected from the BM by lineage depletion and flow cytometry sorting as described in detail previously [39].

Cell culture, transfection, CRISPR/Cas9-mediated miR-125a deletion, and in vitro treatments

U937, THP1, NB4, and GDM-1 cells were maintained at $37{ }^{\circ} \mathrm{C} / 5 \% \mathrm{CO}_{2}$ in RPMI-1640; $293 \mathrm{~T}$ were cultivated the same way in DMEM (all from Sigma-Aldrich). The medium was enriched with $10 \%$ heat-inactivated fetal bovine serum (FBS) and $1 \mathrm{X}$ Antibiotic-Antimycotic 
(Thermo Fischer Scientific, Waltham, MA, USA, including $100 \mathrm{U} / \mathrm{mL}$ penicillin, $100 \mathrm{mg} / \mathrm{mL}$ streptomycin and $0.25 \mathrm{mg} / \mathrm{mL}$ amphotericin B). DMEM medium was further supplemented with 1X GlutaMAX (Thermo Fischer Scientific). For overexpression of miR-125a, THP1 were lentivirally transduced with pEZX-MR03-miR-125a (THP1 miR-125a OE) or the respective empty vector control (THP1 control OE; all constructs from GeneCopoeia, Rockville, MD, USA) according to a previously published protocol [39-41]. Selection of stable cells was performed with $1.5 \mu \mathrm{g} / \mathrm{mL}$ puromycin. For miR-125a knockdown experiments, THP1 cells were transfected with miR-125a-specific miRIDIAN hairpin inhibitors (THP1 miR-125a KD) or scrambled controls (THP1 control KD) using DharmaFECT lipofection as previously described [41, 42] (all reagents from Dharmacon, Lafayette, CO, USA). For generation of miR-125a knockouts in U937 cells, the CRISPR/Cas9 technology was employed using a plasmid-based system with two different singleguide RNAs (sgRNAs) targeting the up- and downstream region of miR-125a. sgRNAs were ligated into pX458 plasmids (Addgene, Watertown, MA, USA; ordering number \#48138), originally generated from the Zhang lab [43], co-expressing Cas9 from Streptococcus pyogenes and EGFP. U937 cells were co-transfected with the generated plasmids using a Neon Nucleofector (Thermo Fischer) according to the manufacturer's instructions, and EGFPpositive single cells were sorted using the FACSAria III (BD biosciences). Successful miR-125a knockout was validated by Sanger sequencing and $\mathrm{qPCR}$ as previously described $[42,44,45]$. Primer and sgRNA sequences are displayed in Additional file 1: Table 2. In vitro treatments were performed with $2.5 \mu \mathrm{M}$ Aza (PeproTech, Rocky Hill, NJ, USA; dissolved in RPMI), $5 \mu \mathrm{M}$ Dec (Sigma-Aldrich; dissolved in RPMI), and $0.5 \mu \mathrm{M}$ staurosporine (Abcam, Cambridge, UK, dissolved in DMSO) as indicated in the respective figures.

\section{miR-microarray and qPCR expression profiling}

RNA isolation was performed using the miRNeasy Micro Kit (Qiagen, Hilden, Germany) according to the manufacturer's protocol. The quality of RNA was controlled on a Bioanalyzer BA2100 (Agilent; Foster City, CA), and only samples with an RNA integrity number (RIN) greater than 9 were taken. For miR-microarray expression profiling in murine HSPCs, 300 ng of RNA was assessed using the Affymetrix GeneChip miRNA 4.0 (Affymetrix, Santa Clara, CA, USA) according to the manufacturer's instructions. Following the miRNA database miRBase (version 20), this kit comprises 36353 gene probes, including 1908 murine miRNAs. Affymetrix Expression Console 1.1.2 was employed for the evaluation of internal array controls and preanalysis, while Partek Genomic Suite v6.6 software (Partek Inc; St Louis, MO) was used for data normalization and analysis. All of these processes have been described in more detail previously [40]. Microarray miR expression data have been deposited in the NCBI's GEO (https://www.ncbi.nlm.nih.gov/geo/) and are accessible through GEO accession number GSE145083. For qPCR experiments, cDNA was generated using the miScript II RT Kit (Qiagen). The expression of miR-125a, miR-150 and miR-26 was assessed on a LightCycler 480 Instrument II (Roche Life Sciences, Penzberg, Germany) using the miScript SYBR Green PCR Kit (Qiagen) and analyzed by employing the $\triangle \Delta C T$ method as previously described [40, 41, 46]. SNORD72, SNORD61, and RNU6b (Qiagen) were used as reference genes. U937 or GDM-1 cells (for all primary human specimens) and untreated/vector-transfected controls, respectively, served as calibrators. Primer sequences are displayed in Additional file 1: Table 2.

\section{Bisulfite sequencing}

Bisulfite conversion from U937 cells was performed using the EpiTect Bisulfite Kit (Qiagen) according to the manufacturer's instructions. For targeted methylation analysis of the micro-RNA-125a upstream region, $2 \mu \mathrm{l}$ of bisulfite-treated DNA was used in a nested PCR approach with the KAPA HiFi HotStart Uracil+Kit (Roche, Mannheim, Germany; for primer sequences see Additional file 1: Table 2). Subsequently, PCR products were indexed and prepared for next-generation sequencing according to published procedures [47, 48]. Libraries were sequenced at $8 \mathrm{pM}$ on an Illumina MiSeq Desktop sequencer with v3 600 chemistry and 20\% PhiX control DNA (Illumina, San Diego, CA, USA) according to manufacturer's instructions. Data were analyzed with the BiQ Analyzer HT software [49].

\section{Analysis of cell growth, proliferation, and apoptosis}

Growth curves were performed as previously described [41, 46]; the number of viable cells was assessed on a TC-20 (Bio-Rad, Hercules, CA, USA). Cell cycle progression/proliferation was assessed by flow cytometry after BrdU/7AAD staining using the BrdU Flow Kit (BD Pharmingen, Franklin Lakes, New Jersey, USA) according to the manufacturer's instructions and as described previously $[41,46,50,51]$. BrdU incubation was performed at a concentration of $50 \mu \mathrm{M}$ for $1 \mathrm{~h}$, and an LSR II machine (BD Biosciences) was used for flow cytometry. Apoptosis was assessed by Annexin-V/7AAD assays (BD Biosciences), as described earlier [41, 46, 50, 51], using a CytoFLEX LX flow cytometer (Beckman Coulter, Brea, CA, USA). 


\section{Statistical analyses and database retrieval}

Methylation $\beta$-values were downloaded via the NCBI's GEO (https://www.ncbi.nlm.nih.gov/geo/) on 25 September 2020 from the publicly available GSE40870 dataset of Klco and coworkers [27]. The changes in methylation $\beta$-values in Dec/cytarabine-treated conditions compared to the respective control-treated conditions were calculated by one-sample $t$ test as outlined in more detail in Figure legend S5.

Group differences in miR-125a expression values of primary patients were compared by Mann-Whitney U or Kruskal-Wallis test. The Wilcoxon signed-rank test was employed for comparison of miR-125a expression before and after HMA treatment in paired patient specimens. Spearman's rank correlation coefficient was used to analyze the correlation of miR-125a with relevant continuous variables within the patient cohort (white blood cell counts, BM blasts, and age). Mouse data and in vitro experiments were analyzed by unpaired, paired, or one-sample $t$ test as depicted in detail in the respective figure legends. All analyses were performed in GraphPad Prism, version 8 (San Diego, CA, USA), and $\mathrm{R}$ version 3.6.1 (https://www.r-project.org/). All tests were performed two-sided, and a $P$ value $<0.050$ was considered statistically significant.

\section{Supplementary information}

Supplementary information accompanies this paper at https://doi. org/10.1186/s13148-020-00979-2.

Additional file 1. Supplementary information.

\section{Abbreviations}

AML: Acute myeloid leukemia; AZA: Azacitidine; BM: Bone marrow; CMML: Chronic myelomonocytic leukemia; DEC: Decitabine; DSMZ: German National Resource Center for Biological Material; FBS: Fetal bovine serum; GEO: Gene Expression Omnibus; HMA: Hypomethylating agent; HR-MDS: High-risk myelodysplastic syndrome; HSC: Hematopoietic stem cell; HSPC: Hematopoietic stem and progenitor cells; LSK: Lin ${ }^{-} / \mathrm{Sca}-1^{+} / \mathrm{C}-\mathrm{Kit}^{+}$cells; MDS/MPN: Myelodysplastic syndrome/myeloproliferative neoplasia overlap syndrome; miR: Micro-RNA; MPD: Myeloproliferative disease; MUG: Medical University of Graz; NCBI: National Center for Biotechnology Information; plpC: Polyinosinic polycytidylic acid; RIN: RNA integrity number; sgRNA: Single-guide RNA; VNTR: Variable number of tandem repeat profiling.

\section{Acknowledgements}

We are thankful to all study participants for sample donation. This project was supported by Biobank Graz.

\section{Authors' contributions}

$J \mathrm{LB}, \mathrm{BP}, \mathrm{SH}, \mathrm{MCM}, \mathrm{SW}, \mathrm{BU}, \mathrm{IK}, \mathrm{ST}$, and AZ performed the research; AR, KG, MP $\mathrm{GH}, \mathrm{HSt}, \mathrm{AW}, \mathrm{HSi}$, and $\mathrm{AZ}$ contributed essential reagents, tools, or patient specimens; JLB, AR, IK, ST, MP, AW, HSi, and AZ analyzed the data; GP, GB, and $A B$ performed the statistical analyzes; JLB and $A Z$ wrote the manuscript; all authors read the manuscript and/or revised it critically; $A Z$ designed the research study; all authors approved the submitted and final version. All authors read and approved the final manuscript.

\section{Funding}

This study was supported by research funding from the Austrian Society of Internal Medicine (Joseph-Skoda Awards to A. Zebisch and A. Reinisch) and the Austrian Science Fund (grants P 31430-458 B26 to H. Sill and P32783 to A. Reinisch). Research in the laboratories of A. Zebisch, A. Wölfler, and H. Sill is further supported by Leukämiehilfe Steiermark. A. Reinisch was also supported by the Austrian Society of Hematology and Medical Oncology (Clinical Research Grant) and MEFOgraz. PhD candidate J.L. Berg received funding from the MUG within the PhD program Molecular Medicine.

\section{Availability of data and materials}

Microarray miR expression data have been deposited in the NCB's GEO (https ://www.ncbi.nlm.nih.gov/geo/) and are accessible through GEO accession number GSE145083. All other data are available from the corresponding author on reasonable request.

\section{Ethics approval and consent to participate}

The study was approved by the institutional review board of the MUG (EK 28-481 ex 15/16) and was conducted in accordance with the Declaration of Helsinki. All mouse experiments were approved by the Austrian Federal Ministry for Science, Research and Economy (GZ: BMWF-66.010/0050-II/3b/2013).

\section{Competing interests}

The authors declare that they have no competing interests.

\section{Author details}

${ }^{1}$ Division of Hematology, Medical University of Graz, Auenbruggerplatz 38, 8036 Graz, Austria. ${ }^{2}$ Core Facility Molecular Biology, Medical University of Graz, Graz, Austria. ${ }^{3}$ Department of Genetics, University of Saarland, Saarbrücken, Germany. ${ }^{4}$ Institute for Medical Informatics, Statistics and Documentation, Medical University of Graz, Graz, Austria. ${ }^{5}$ 5th Medical Department with Hematology, Oncology and Palliative Medicine, Hospital Hietzing, Vienna, Austria. ${ }^{6}$ Sigmund Freud University, Vienna, Austria. ${ }^{7}$ Division of Oncology, Medical University of Graz, Graz, Austria. ${ }^{8}$ Department of Experimental Therapeutics, The University of Texas MD Anderson Cancer Centre, Houston, TX, USA. ${ }^{9}$ Diagnostic and Research Institute of Pathology, Medical University of Graz, Graz, Austria. ${ }^{10}$ Otto Loewi Research Centre, Immunology and Pathophysiology, Medical University of Graz, Graz, Austria. ${ }^{11}$ Otto-Loewi Research Centre for Vascular Biology, Immunology and Inflammation, Division of Pharmacology, Medical University of Graz, Universitätsplatz 4, 8010 Graz, Austria.

Received: 3 June 2020 Accepted: 17 November 2020

Published online: 06 January 2021

\section{References}

1. Arber DA, Orazi A, Hasserjian R, Thiele J, Borowitz MJ, Le Beau MM, et al. The 2016 revision to the World Health Organization classification of myeloid neoplasms and acute leukemia. Blood. 2016;127:2391-405.

2. Itzykson R, Fenaux P, Bowen D, Cross NCP, Cortes J, De Witte T, et al. Diagnosis and treatment of chronic myelomonocytic leukemias in adults: recommendations from the European Hematology Association and the European LeukemiaNet. Hemasphere. 2018;2:e150

3. Patnaik MM, Tefferi A. Chronic Myelomonocytic leukemia: 2020 update on diagnosis, risk-stratification and management. Am J Hematol. 2020:95:97-115.

4. Geissler K, Jager E, Barna A, Gurbisz M, Marschon R, GrafT, et al. The Austrian biodatabase for chronic myelomonocytic leukemia (ABCMML): a representative and useful real-life data source for further biomedical research. Wien Klin Wochenschr. 2019;131:410-8.

5. Diesch J, Zwick A, Garz AK, Palau A, Buschbeck M, Gotze KS. A clinicalmolecular update on azanucleoside-based therapy for the treatment of hematologic cancers. Clin Epigenetics. 2016;8:71016-0237-y. eCollection 2016

6. Zebisch A, Hatzl S, Pichler M, Wolfler A, Sill H. Therapeutic resistance in acute myeloid leukemia: the role of non-coding RNAs. Int J Mol Sci. 2016;17:E2080.

7. Fabbri M, Croce CM, Calin GA. MicroRNAs. Cancer J. 2008;14:1-6.

8. Zebisch A, Caraffini V, Sill H. RAF kinase inhibitor protein in myeloid leukemogenesis. Int J Mol Sci. 2019;20:https://doi.org/10.3390/ijms20225756. 
9. Croce CM. Causes and consequences of microRNA dysregulation in cancer. Nat Rev Genet. 2009;10:704-14.

10. Calin GA, Croce CM. MicroRNA signatures in human cancers. Nat Rev Cancer. 2006;6:857-66.

11. Yendamuri S, Calin GA. The role of microRNA in human leukemia: a review. Leukemia. 2009;23:1257-63.

12. Gordon JE, Wong JJ, Rasko JE. MicroRNAs in myeloid malignancies. Br J Haematol. 2013;162:162-76.

13. Braun BS, Tuveson DA, Kong N, Le DT, Kogan SC, Rozmus J, et al. Somatic activation of oncogenic Kras in hematopoietic cells initiates a rapidly fatal myeloproliferative disorder. Proc Natl Acad Sci USA. 2004;101:597-602.

14. Chan IT, Kutok JL, Williams IR, Cohen S, Kelly L, Shigematsu H, et al. Conditional expression of oncogenic $K$-ras from its endogenous promoter induces a myeloproliferative disease. J Clin Invest. 2004;113:528-38.

15. Itzykson R, Kosmider O, Renneville A, Morabito M, Preudhomme C, Berthon C, et al. Clonal architecture of chronic myelomonocytic leukemias. Blood. 2013;121:2186-98.

16. Tatsumi N, Hojo N, Yamada O, Ogawa M, Katsura Y, Kawata S, et al. Deficiency in WT1-targeting microRNA-125a leads to myeloid malignancies and urogenital abnormalities. Oncogene. 2016;35:1003-14.

17. Fang ZH, Wang SL, Zhao JT, Lin ZJ, Chen LY, Su R, et al. miR-150 exerts antileukemia activity in vitro and in vivo through regulating genes in multiple pathways. Cell Death Dis. 2016;7:e2371.

18. Jiang Q, Isquith J, Zipeto MA, Diep RH, Pham J, Delos Santos N, et al. Hyper-Editing of cell-cycle regulatory and tumor suppressor RNA promotes malignant progenitor propagation. Cancer Cell. 2019;35(81):94.e7.

19. Salvatori B, losue I, Djodji Damas N, Mangiavacchi A, Chiaretti S, Messina $M$, et al. Critical role of C-Myc in acute myeloid leukemia involving direct regulation of miR-26a and histone methyltransferase EZH2. Genes Cancer. 2011;2:585-92.

20. Chen J, Ellison FM, Keyvanfar K, Omokaro SO, Desierto MJ, Eckhaus MA, et al. Enrichment of hematopoietic stem cells with SLAM and LSK markers for the detection of hematopoietic stem cell function in normal and Trp53 null mice. Exp Hematol. 2008;36:1236-43.

21. Gerrits $A$, Walasek MA, Olthof $S$, Weersing E, Ritsema M, Zwart E, et al. Genetic screen identifies microRNA cluster 99b/let-7e/125a as a regulator of primitive hematopoietic cells. Blood. 2012;1 19:377-87.

22. Chen $\mathrm{H}, \mathrm{Xu} \mathrm{Z}$. Hypermethylation-associated silencing of miR-125a and miR-125b: a potential marker in colorectal cancer. Dis Markers. 2015:2015:345080.

23. Ufkin ML, Peterson S, Yang X, Driscoll H, Duarte C, Sathyanarayana P. miR125a regulates cell cycle, proliferation, and apoptosis by targeting the ErbB pathway in acute myeloid leukemia. Leuk Res. 2014;38:402-10.

24. Liu H, Ma Y, Liu C, Li P, Yu T. Reduced miR-125a-5p level in non-smallcell lung cancer is associated with tumour progression. Open Biol. 2018;8:https://doi.org/10.1098/rsob.180118.

25. Cai M, Chen Q, Shen J, Lv C, Cai L. Epigenetic silenced miR-125a-5p could be self-activated through targeting Suv39H1 in gastric cancer. J Cell Mol Med. 2018:22:4721-31.

26. Savona MR, Malcovati L, Komrokji R, Tiu RV, Mughal TI, Orazi A, et al. An international consortium proposal of uniform response criteria for myelodysplastic/myeloproliferative neoplasms (MDS/MPN) in adults. Blood. 2015;125:1857-65.

27. Klco JM, Spencer DH, Lamprecht TL, Sarkaria SM, Wylie T, Magrini V, et al. Genomic impact of transient low-dose decitabine treatment on primary AML cells. Blood. 2013;121:1633-43.

28. Flotho C, Claus R, Batz C, Schneider M, Sandrock I, Ihde S, et al. The DNA methyltransferase inhibitors azacitidine, decitabine and zebularine exert differential effects on cancer gene expression in acute myeloid leukemia cells. Leukemia. 2009;23:1019-28.

29. Imanishi S, Umezu T, Ohtsuki K, Kobayashi C, Ohyashiki K, Ohyashiki $\mathrm{JH}$. Constitutive activation of the ATM/BRCA1 pathway prevents DNA damage-induced apoptosis in 5-azacytidine-resistant cell lines. Biochem Pharmacol. 2014:89:361-9.

30. Shaham L, Binder V, Gefen N, Borkhardt A, Izraeli S. MiR-125 in normal and malignant hematopoiesis. Leukemia. 2012;26:2011-8.

31. Guo S, Bai H, Megyola CM, Halene S, Krause DS, Scadden DT, et al. Complex oncogene dependence in microRNA-125a-induced myeloproliferative neoplasms. Proc Natl Acad Sci USA. 2012;109:16636-41.

32. Li Z, Chen P, Su R, Li Y, Hu C, Wang Y, et al. Overexpression and knockout of miR-126 both promote leukemogenesis. Blood. 2015;126:2005-15.
33. Solly F, Koering C, Mohamed AM, Maucort-Boulch D, Robert G, Auberger $P$, et al. An miRNA-DNMT1 axis is involved in azacitidine resistance and predicts survival in higher-risk myelodysplastic syndrome and low blast count acute myeloid leukemia. Clin Cancer Res. 2017;23:3025-34.

34. Christopher AF, Kaur RP, Kaur G, Kaur A, Gupta V, Bansal P. MicroRNA therapeutics: discovering novel targets and developing specific therapy. Perspect Clin Res. 2016;7:68-74.

35. Geissler K, Jager E, Barna A, Gurbisz M, Graf T, Graf E, et al. Correlation of RAS-pathway mutations and spontaneous myeloid colony growth with progression and transformation in chronic myelomonocytic leukemia-A retrospective analysis in 337 patients. Int J Mol Sci. 2020;21:https://doi. org/10.3390/ijms21083025.

36. Lal R, Lind K, Heitzer E, Ulz P, Aubell K, Kashofer K, et al. Somatic TP53 mutations characterize preleukemic stem cells in acute myeloid leukemia. Blood. 2017;129:2587-91.

37. Zebisch A, Lal R, Muller M, Lind K, Kashofer K, Girschikofsky M, et al. Acute myeloid leukemia with TP53 germ line mutations. Blood. 2016:128:2270-2.

38. Prochazka KT, Pregartner G, Rucker FG, Heitzer E, Pabst G, Wolfler A, et al. Clinical implications of subclonal TP53 mutations in acute myeloid leukemia. Haematologica. 2019;104:516-23.

39. Caraffini V, Geiger O, Rosenberger A, Hatzl S, Perfler B, Berg JL, et al. Loss of RAF kinase inhibitor protein is involved in myelomonocytic differentiation and aggravates RAS-driven myeloid leukemogenesis. Haematologica. 2020;105:375-86.

40. Caraffini V, Perfler B, Berg JL, Uhl B, Schauer S, Kashofer K, et al. Loss of RKIP is a frequent event in myeloid sarcoma and promotes leukemic tissue infiltration. Blood. 2018;131:826-30.

41. Hatzl S, Geiger O, Kuepper MK, Caraffini V, Seime T, Furlan T, et al. Increased expression of miR-23a mediates a loss of expression in the RAF kinase inhibitor protein RKIP. Cancer Res. 2016;76:3644-54.

42. Zebisch A, Haller M, Hiden K, Goebel T, Hoefler G, Troppmair J, et al. Loss of RAF kinase inhibitor protein is a somatic event in the pathogenesis of therapy-related acute myeloid leukemias with C-RAF germline mutations. Leukemia. 2009;23:1049-53.

43. Ran FA, Hsu PD, Wright J, Agarwala V, Scott DA, Zhang F. Genome engineering using the CRISPR-Cas9 system. Nat Protoc. 2013;8:2281-308.

44. Zebisch A, Cerroni L, Beham-Schmid C, Sill H. Therapy-related leukemia cutis: case study of an aggressive disorder. Ann Hematol. 2003;82:705-7.

45. Zebisch A, Staber PB, Delavar A, Bodner C, Hiden K, Fischereder K, et al. Two transforming C-RAF germ-line mutations identified in patients with therapy-related acute myeloid leukemia. Cancer Res. 2006;66:3401-8.

46. Zebisch A, Wolfler A, Fried I, Wolf O, Lind K, Bodner C, et al. Frequent loss of RAF kinase inhibitor protein expression in acute myeloid leukemia. Leukemia. 2012;26:1842-9.

47. Klymiuk I, Bilgilier C, Stadlmann A, Thannesberger J, Kastner MT, Hogenauer $C$, et al. The human gastric microbiome Is predicated upon infection with helicobacter pylori. Front Microbiol. 2017;8:2508.

48. Gries J, Schumacher D, Arand J, Lutsik P, Markelova MR, Fichtner I, et al. Bi-PROF: bisulfite profiling of target regions using 454 GS FLXTitanium technology. Epigenetics. 2013;8:765-71.

49. Lutsik P, Feuerbach L, Arand J, Lengauer T, Walter J, Bock C. BiQ Analyzer HT: locus-specific analysis of DNA methylation by high-throughput bisulfite sequencing. Nucleic Acids Res. 2011;39:W551-6.

50. Zebisch A, Schulz E, Grosso M, Lombardo B, Acierno G, Sill H, et al. Identification of a novel variant of epsilon-gamma-delta-beta thalassemia highlights limitations of next generation sequencing. Am J Hematol. 2015;90:E52-4

51. Schulz E, Klampfl P, Holzapfel S, Janecke AR, Ulz P, Renner W, et al. Germline variants in the SEMA4A gene predispose to familial colorectal cancer type X. Nat Commun. 2014;5:5191.

52. Potenza N, Panella M, Castiello F, Mosca N, Amendola E, Russo A. Molecular mechanisms governing microRNA-125a expression in human hepatocellular carcinoma cells. Sci Rep. 2017;7:10712,017-11418-3.

\section{Publisher's Note}

Springer Nature remains neutral with regard to jurisdictional claims in published maps and institutional affiliations. 